\title{
Screening of jute and kenaf varieties for salinity tolerance
}

\author{
Md. Isfatuzzaman Bhuyan ${ }^{2}$, K. M. Mehadi Hassan ${ }^{2}$, Nowrose Jahan Lipi ${ }^{2}$, Md. Rafiq Uddin ${ }^{1}$, \\ Md. Monirul Islam ${ }^{1 *}$, Mahbuba Ferdous ${ }^{1}$, Nazmul Hasan Antor ${ }^{2}$, Parna Das $^{2}$ \\ ${ }^{1}$ Department of Soil Science, Patuakhali Science and Technology University, Dumki, Patuakhali, Dhaka, Bangladesh \\ ${ }^{2}$ Department of Agronomy, Patuakhali Science and Technology University, Dumki, Patuakhali, Dhaka, Bangladesh \\ *Corresponding author E-mail: monirulpstu1@gmail.com
}

\begin{abstract}
A study was conducted in the Department of Agronomy, Bangladesh Agricultural University (BAU), Mymensingh from April to August 2012 to examine the salinity tolerance of eight jute varieties (CVE-3, C-83, CVL-1, BJC-7370, O-795, O-9897, OM-1, O-72) and two kenaf varieties (HC-95 and HC-2). Initially germination of these varieties were evaluated under six salinity levels viz. $0 \mathrm{mM}, 20 \mathrm{mM}, 40 \mathrm{mM}$, $60 \mathrm{mM}, 80 \mathrm{mM}$, and $100 \mathrm{mM} \mathrm{NaCl}$ in the seed laboratory of the Department of Agronomy. Afterwards, all the varieties was grown in pots in the net house under four salinity levels viz. $0 \mathrm{mM}, 25 \mathrm{mM}, 50 \mathrm{mM}$, and $75 \mathrm{mM}$. The results from the germination study revealed that under control condition $(0 \mathrm{mM} \mathrm{NaCl})$ all the jute varieties showed germination more than $80 \%$ both at 7 and at 14 days after seed sowing, whereas kenaf varieties had germination a little less than $80 \%$. Among the jute varieties, O-72 showed the highest germination (92\%), which was statistically similar with those of OM-1(91\%), O-795 (90\%), and C-83(87\%). Salinity stress decreased germination drastically in all of the jute and kenaf varieties. A salinity level of $100 \mathrm{mM}$ caused the highest germination inhibition (74.70\%) in jute variety CVL-1, which was very close to those of BJC-7370 and O-72. On the other hand, the lowest germination inhibition (51.11\%) was recorded in jute variety O795. The results of the pot trail showed that the plant characters of jute and kenaf varieties were affected significantly by salinity stress. All the varieties produced their respective plant height, number of leaves per plant, and plant dry weight under control condition (no salinity). Among these varieties, CVE-3 produced the highest plant height $(145.2 \mathrm{~cm})$, and total dry weight $(22.55 \mathrm{~g})$, whereas O-72 produced the highest number of leaves per plant (24.67). All these plant characters decreased sharply due to salinity stress irrespective of variety. However, the rate of decrease of plant characters occurred differentially in the jute and kenaf varieties. The highest rate of decrease in number of leaves $(74.22 \%)$ was found from the variety CVE-3, whereas the lowest one was recorded from the variety HC-2 (51.68\%). Salinity stress caused the highest decrease in total plant dry weight $(73.68 \%)$ in the variety CVL-1 and plant height (73.64\%) also in the variety CVL-1. On the contrary, the lowest decrease in plant dry weight $(50.99 \%)$ was found in the variety O-9897 and plant height also in variety O-9897 (50.88\%). Based on the results from germination test and pot trail, it can be inferred that jute variety O-9897 appeared to be the most salt tolerant followed by O-795, HC-2, HC-95, CVE-3, O-72, C-83, BJC-7370, OM-1, and CVL-1.
\end{abstract}

Keywords: Jute; KENAF; Saline Soil; Bangladesh

\section{Introduction}

Jute and kenaf are important fibre crops which belong to the family Tiliaceae. Two cultivated species of jute are Corchorus capsularis (Deshi jute) and Corchorus olitorius (Tossa jute). The commercial product of the fibre is derived from these two species of genus Corchorus, among 40 species distributed throughout the tropics (Alim, 1978). Jute is the second important fibre crop of the world (Saha, 1996). It is generally known as golden fibre of Bangladesh as it contributes significantly to the economy. Jute fibre is used for making gunny bags, sacking, carpet backing cloths, mats blankets, furnishing fabrics and packing materials as an alternative to polyethyline bags. It is also used as micro-crystal cellulose in pharmaceutical laboratories and jute Zeo-Textice for the constructions. In addition, jute fibre is a good source of different grades of paper pulp (Akhtaruzzaman and Shafi, 1995; Jahan M.S.2001). Salinity is one of the major widespread environmental stresses that can limit growth and development of salt sensitive plant (Greenway and Munns, 1980). The salinity developed in soil adversely affects the growth and yield of different crop plants. Seed germination is affected by the increase in salinity (Purhpan and Rangasamy, 2002). Crop plants show reduction in dry matter accumulation and grain yield under salt stress, which is invariably accompanied with pronounced changes in their ionic composition. The salinity damage manifests most prominently in the dry season when is caused by evaporation. There adverse physiological effects of salinity may be attributed to nonavailability of water (Singh et al. 2001), reduction in photosynthesis through loss of turgidity (Gufran 1994), impeded nutrient uptake causing deficiency and ion toxicity to plant (Varshney et al. 1998). Selective uptake of non-toxic ions into the vascular saps and compartmentalization of toxic ions into the older plant parts is considered to be important basis of tolerance to salinity. Millions of hectares of land throughout the world are too saline to produce economic crops and more land is becoming nonproductive each year because of salt accumulation. In recent years, requirement for jute and kenaf fiber has tremendously increased in Bangladesh. Jute can grow readily in saline soils (Ma et al., 2009). Considering its tolerance especially to the chlorine salinity, jute has been recently suggested as a promising candidate for planting in wetlands and saline soils in Bangladesh. 


\section{Materials and methods}

A preliminary germination test was conducted in the laboratory of the Department of Agronomy, Bangladesh Agricultural University (BAU), Mymensingh during the period from 15 April, 2012 to 30 April 2012 for evaluating the rate of germination of jute and kenaf cultivars for salinity tolerance.

Description of the experimental site

i) Location

The growth performance was carried out at the net house of the Department of Agronomy, Bangladesh Agricultural University, Mymensingh. Experimental site was located at $24^{\circ} \mathrm{N}$ latitude and $94^{\circ} \mathrm{E}$ longitude of 18 meter from the sea level. The experimental site belongs to the Agro-ecological Zone of the Old Brahmaputra Floodplain (AEZ 9).

ii) Soil

The soil was collected within $6 \mathrm{~cm}$, from the top soil of the Agronomy Field Laboratory, Bangladesh Agricultural University, Mymensingh. The soil covers a large area of the Brahmaputra River born sediments which was laid down before the river shifted into its Jamuna Channel about 200 years ago (UNDP and FAO, 1998). The soil of the experimental pot belongs to the Sonatola Series of noncalcareous dark grey floodplain soil type under the Old Brahmaputra Alluvial Tract. The experimental soil was loamy in texture having a soil $\mathrm{pH}$ value of 6.43 , moderate in organic matter content (Appendix I).

iii) Climate

The experimental area was under the sub-tropical climate that is characterized by high temperature, high humidity and heavy rainfall with occasional gusty winds in kharif season (April- September) and less rainfall associated with moderately low temperature during the rabi season (October-March). Weather information regarding temperature, relative humidity, rainfall and sunshine hours prevailed at the experimental site during the cropping season (March to July) of the study.

Treatments of the experiment

Ten varieties of jute and kenaf and six levels of salinity used in the experiment were as follows:
a) Variety-Ten
01) CVE-3 02) C-83
03) CVL-1 04) BJC-7370
05) O-795 06) O-9897
07) OM-1 08) O-72
09) HC-95 10) HC-2
b) Level of Salinity (mM)-Six
i) $0 \mathrm{mM} \mathrm{NaCl}(\mathrm{So})$
ii) $20 \mathrm{mM} \mathrm{NaCl}\left(\mathrm{S}_{1}\right)$
iii) $40 \mathrm{mM} \mathrm{NaCl}\left(\mathrm{S}_{2}\right)$
iv) $60 \mathrm{mM} \mathrm{NaCl}\left(\mathrm{S}_{3}\right)$
v) $80 \mathrm{mM} \mathrm{NaCl}\left(\mathrm{S}_{4}\right)$
vi) $100 \mathrm{mM} \mathrm{NaCl}\left(\mathrm{S}_{5}\right)$

Design and layout of the experiment

The experiment was laid out in a Randomized Complete Block Design (RCBD) with three replications. The total number of pots used in this study was $120(10 \times 4 \times 3)$. Each plastic pot was $15 \mathrm{~cm}$ deep with $17 \mathrm{~cm}$ diameter at the top. Top surface of each pot measured $0.22 \mathrm{~m}^{2}$. Each pot contained five plants and denoted a replication. Considerable spacing was maintained among pots for convenience of cultural operations.

Jute and kenaf

The varieties used for this experiment were CVE-3, C-83, CVL-1, BJC-7370, O-795, O-9897, OM-1, O-72, HC-95, and HC-2.These varieties was developed by Bangladesh Jute Research Institute (BJRI). Some important description of these varieties are as follows:

CVE-3

Released year: 1977

Plant type: Stem green, bright coppery red on older branches, upper portion of the petiole and stipulate bright coppery red. Leaf ovate lancelets, seeds chocolate brown.
Special feature: Quick growing variety, early maturing, suitable for early sown area.

C-83

Released year: 1979

Plant type: Stem green, upper portion of the petiole coppery red, leaves nearly ovate, seeds small with light brown color.

Special feature: Short day insensitive, no risk of premature flowering, very wider time for sowing.

CVL-1

Released year: 1977

Plant type: Full green, stipule green, petiole green, leaf ovate lanceolate, light coppery red on old branches.

Special feature: Most tolerate to mosaic virus, suitable for late sown area.

BJC-7370

Released year: 2007

Plant type: Stem green tall, unbranched, leaves lanceolate, petiole green, seed is blue in color.

Special feature: Short day and low temperature tolerance, perform better than existing varieties C-83 and CVE-3 at farm level.

O-9897

Released year: 1987

Plant type: Fully deep green, unbranched, leaves ovate deep green, succulent, seeds brown with green tinage on seed coat.

Special feature: Short day insensitive, no risk of premature flowering, very wider time for sowing.

OM-1

Released year: 1995

Plant type: Stem tall, cylindrical and deep green, leaves ovate, and seeds brownish.

Special feature: Less photosensitive, suitable for early sowing.

O-72

Released year: 2002

Plant type: Stem tall, unbranched, cylindrical and green,seeds brownish gray.

Special feature: Less photosensitive, suitable for early sowing. HC-95

Released year: 1995

Plant type: Stem tall cylindrical, unbranched, whole plant deep green, leaves lobed, seeds subreniform grayish brown.

Special feature: suitable for medium low, less fertile soil and flood affected land.

HC-2

Released year: 1977

Plant type: Stem green pigmented and petiole green, leaves simple cordate, petiole longer than lamina, seeds subreniform grayish brown.

Special feature: suitable for medium low, less fertile soil and flood affected land.

\section{Results and discussion}

Results obtained from the study to screen out the salt tolerant jute and kenaf varieties under salinity stress condition. To strengthen the discussion, information in the form of tables and graphs are provided. The germination (percentage), plant characteristics and yield of jute and kenaf in relation to salinity level and varieties were found significant. Germination (percentage) of jute and kenaf variety under different salinity levels. 
Table 1: Interaction Effects of Variety and Salinity Level on Germination Percentage

\begin{tabular}{|c|c|c|c|c|}
\hline $\begin{array}{l}\text { Variety and sa- } \\
\text { linity }\end{array}$ & $\%$ Germination & & & \\
\hline & $\begin{array}{l}7 \text { DAS (Days After } \\
\text { Sowing) }\end{array}$ & $\begin{array}{l}\text { Decrease in germination(\%) due to } \\
\text { salinity stress }\end{array}$ & $\begin{array}{l}14 \text { DAS (Days After } \\
\text { Sowing) }\end{array}$ & $\begin{array}{l}\text { Decrease in germination(\%) due to } \\
\text { salinity stress }\end{array}$ \\
\hline $\mathrm{V}_{1} \mathrm{~S}_{0}$ & $84.00 \mathrm{de}$ & 0 & $84.00 \mathrm{de}$ & 0 \\
\hline $\mathrm{V}_{1} \mathrm{~S}_{1}$ & $75.00 \mathrm{~g}$ & 10.71 & $45.00 \mathrm{~g}$ & 46.42 \\
\hline $\mathrm{V}_{1} \mathrm{~S}_{2}$ & $57.00 \mathrm{ijkl}$ & 32.14 & 38.00 hij & 54.76 \\
\hline $\mathrm{V}_{1} \mathrm{~S}_{3}$ & 43.75 qrs & 49.10 & $34.50 \mathrm{jkl}$ & 58.92 \\
\hline $\mathrm{V}_{1} \mathrm{~S}_{4}$ & $33.00 \mathrm{vw}$ & 60.71 & 26.50 no & 68.45 \\
\hline $\mathrm{V}_{1} \mathrm{~S}_{5}$ & $23.75 \mathrm{x}$ & 72.91 & $11.00 \mathrm{vwx}$ & 86.90 \\
\hline $\mathrm{V}_{2} \mathrm{~S}_{0}$ & $87.00 \mathrm{abcd}$ & 0 & $87.00 \mathrm{bcd}$ & 0 \\
\hline $\mathrm{V}_{2} \mathrm{~S}_{1}$ & $58.50 \mathrm{ijk}$ & 32.75 & $37.50 \mathrm{ij}$ & 56.89 \\
\hline $\mathrm{V}_{2} \mathrm{~S}_{2}$ & 52.0 lmnop & 40.22 & $23.00 \mathrm{opq}$ & 73.56 \\
\hline $\mathrm{V}_{2} \mathrm{~S}_{3}$ & $49.00 \mathrm{opq}$ & 43.67 & 20.00 pqrs & 77.01 \\
\hline $\mathrm{V}_{2} \mathrm{~S}_{4}$ & $47.00 \mathrm{pqr}$ & 40.00 & $18.00 \mathrm{rst}$ & 79.31 \\
\hline $\mathrm{V}_{2} \mathrm{~S}_{5}$ & $36.00 \mathrm{v}$ & 58.62 & 10.00 wxy & 88.50 \\
\hline $\mathrm{V}_{3} \mathrm{~S}_{0}$ & $85.0 \mathrm{cde}$ & 0 & $85.00 \mathrm{de}$ & 0 \\
\hline $\mathrm{V}_{3} \mathrm{~S}_{1}$ & $62.00 \mathrm{hi}$ & 27.05 & $36.00 \mathrm{j}$ & 57.64 \\
\hline $\mathrm{V}_{3} \mathrm{~S}_{2}$ & 50.50 nop & 40.58 & $27.00 \mathrm{mno}$ & 68.23 \\
\hline $\mathrm{V}_{3} \mathrm{~S}_{3}$ & $44.00 \mathrm{qrs}$ & 48.23 & 22.50 opqr & 73.52 \\
\hline $\mathrm{V}_{3} \mathrm{~S}_{4}$ & $33.50 \mathrm{vw}$ & 60.58 & $18.00 \mathrm{rst}$ & 78.82 \\
\hline $\mathrm{V}_{3} \mathrm{~S}_{5}$ & $21.50 \mathrm{x}$ & 74.70 & $8.000 \mathrm{xyz}$ & 90.58 \\
\hline $\mathrm{V}_{4} \mathrm{~S}_{0}$ & 86.00 bcde & 0 & $86.00 \mathrm{~cd}$ & 0 \\
\hline $\mathrm{V}_{4} \mathrm{~S}_{1}$ & $56.50 \mathrm{ijklm}$ & 34.70 & $30.50 \mathrm{lmn}$ & 64.53 \\
\hline $\mathrm{V}_{4} \mathrm{~S}_{2}$ & 52.00 lmnop & 39.53 & 24.00 op & 72.09 \\
\hline $\mathrm{V}_{4} \mathrm{~S}_{3}$ & $42.00 \mathrm{rst}$ & 51.16 & 21.00 pqrs & 75.58 \\
\hline $\mathrm{V}_{4} \mathrm{~S}_{4}$ & 37.00 tuv & 56.97 & $17.00 \mathrm{stu}$ & 80.23 \\
\hline $\mathrm{V}_{4} \mathrm{~S}_{5}$ & $22.00 \mathrm{x}$ & 74.41 & 9.000 wxyz & 89.53 \\
\hline $\mathrm{V}_{5} \mathrm{~S}_{0}$ & $90.00 \mathrm{abc}$ & 0 & $90.00 \mathrm{abc}$ & 0 \\
\hline $\mathrm{V}_{5} \mathrm{~S}_{1}$ & $72.50 \mathrm{~g}$ & 19.45 & $36.00 \mathrm{j}$ & 60.00 \\
\hline $\mathrm{V}_{5} \mathrm{~S}_{2}$ & $57.00 \mathrm{ijkl}$ & 36.67 & $26.00 \mathrm{o}$ & 71.11 \\
\hline $\mathrm{V}_{5} \mathrm{~S}_{3}$ & $53.00 \mathrm{klmno}$ & 41.12 & 21.00 pqrs & 76.67 \\
\hline $\mathrm{V}_{5} \mathrm{~S}_{4}$ & $49.00 \mathrm{opq}$ & 45.56 & 15.00 tuv & 83.34 \\
\hline $\mathrm{V}_{5} \mathrm{~S}_{5}$ & 44.00 qrs & 51.11 & $6.000 \mathrm{yz}$ & 93.34 \\
\hline V6S0 & $83.00 \mathrm{de}$ & 0 & $83.00 \mathrm{de}$ & 0 \\
\hline V6S1 & $58.00 \mathrm{ijk}$ & 30.12 & 38.00 hij & 54.22 \\
\hline V6S2 & $56.00 \mathrm{jklmn}$ & 32.53 & $26.00 \mathrm{o}$ & 68.67 \\
\hline V6S3 & 51.00 mnop & 38.55 & $17.00 \mathrm{stu}$ & 79.52 \\
\hline V6S4 & 44.00 qrs & 46.98 & $13.00 \mathrm{uvw}$ & 83.34 \\
\hline V6S5 & $35.00 \mathrm{vw}$ & 57.80 & 11.000 vwx & 86.74 \\
\hline V7S0 & $91.00 \mathrm{ab}$ & 0 & $91.00 \mathrm{ab}$ & 0 \\
\hline V7S1 & $66.00 \mathrm{~h}$ & 27.57 & $35.00 \mathrm{jk}$ & 61.53 \\
\hline V7S2 & $57.00 \mathrm{ijkl}$ & 37.36 & $26.00 \mathrm{o}$ & 71.42 \\
\hline V7S3 & 51.00 mnop & 43.95 & 19.00 qrst & 79.12 \\
\hline V7S4 & 45.00 qrs & 50.54 & 10.00 wxy & 89.01 \\
\hline V7S5 & $33.00 \mathrm{vw}$ & 60.73 & $4.000 \mathrm{z}$ & 95.60 \\
\hline V8S0 & $92.00 \mathrm{a}$ & 0 & $92.00 \mathrm{a}$ & 0 \\
\hline V8S1 & $61.00 \mathrm{hij}$ & 33.69 & $31.00 \mathrm{klm}$ & 66.30 \\
\hline V8S2 & 51.00 mnop & 44.56 & $23.00 \mathrm{opq}$ & 75.00 \\
\hline V8S3 & $42.00 \mathrm{rst}$ & 54.54 & 13.00 uvw & 85.86 \\
\hline V8S4 & $36.00 \mathrm{uv}$ & 60.86 & 9.000 wxyz & 90.21 \\
\hline V8S5 & $25.00 \mathrm{x}$ & 72.82 & $3.000 \mathrm{z}$ & 96.73 \\
\hline V9S0 & 81.00 ef & 0 & $81.00 \mathrm{e}$ & 0 \\
\hline V9S1 & $59.00 \mathrm{ij}$ & 27.16 & $41.00 \mathrm{ghi}$ & 49.38 \\
\hline V9S2 & 51.00 nop & 37.03 & $24.00 \mathrm{op}$ & 70.37 \\
\hline V9S3 & $43.00 \mathrm{rs}$ & 46.91 & $13.00 \mathrm{uvw}$ & 83.95 \\
\hline V9S4 & $35.00 \mathrm{vw}$ & 56.79 & 9.000 wxyz & 88.89 \\
\hline V9S5 & $22.50 x$ & 72.22 & $2.000 \mathrm{z}$ & 97.53 \\
\hline V10S0 & $77.00 \mathrm{fg}$ & 0 & $77.00 \mathrm{f}$ & 0 \\
\hline V10S1 & $65.00 \mathrm{~h}$ & 15.58 & $42.00 \mathrm{gh}$ & 45.46 \\
\hline V10S2 & $57.00 \mathrm{ijkl}$ & 25.97 & $34.00 \mathrm{jkl}$ & 55.85 \\
\hline V10S3 & 51.00 nop & 33.76 & 21.00 pqrs & 72.72 \\
\hline V10S4 & $41.00 \mathrm{stu}$ & 46.75 & $11.00 \mathrm{vwx}$ & 85.71 \\
\hline V10S5 & $30.00 \mathrm{w}$ & 61.03 & $3.500 \mathrm{z}$ & 95.45 \\
\hline LSD0.05 & 4.73 & & 3.92 & \\
\hline $\begin{array}{l}\text { Level of signifi- } \\
\text { cance }\end{array}$ & $* *$ & & $* *$ & \\
\hline $\mathrm{CV} \%$ & 6.38 & & 8.86 & \\
\hline
\end{tabular}

In a column, figures with common letters or without letter do not differ significantly, whereas figures with dissimilar letters differ significantly (as per DMRT)

** = Significant at $1 \%$ level of probability

$\mathrm{V}_{1}=\mathrm{CVE}-3 \mathrm{~S}_{0}=0 \mathrm{mM} \mathrm{NaCl}$

$\mathrm{V}_{2}=\mathrm{C}-83 \mathrm{~S}_{1}=20 \mathrm{mM} \mathrm{NaCl}$
$\mathrm{V}_{3}=\mathrm{CVL}-1 \mathrm{~S}_{2}=40 \mathrm{mM} \mathrm{NaCl}$

$\mathrm{V}_{4}=\mathrm{BJC}-7370 \mathrm{~S}_{3}=60 \mathrm{mM} \mathrm{NaCl}$

$\mathrm{V}_{5}=0-795 \mathrm{~S}_{4}=80 \mathrm{mM} \mathrm{NaCl}$

$\mathrm{V}_{6}=0-9897 \mathrm{~S}_{5}=100 \mathrm{mM} \mathrm{NaCl}$

$\mathrm{V}_{7}=\mathrm{OM}-1$ 
$\mathrm{V}_{8}=\mathrm{O}-72$

$\mathrm{V}_{9}=\mathrm{HC}-95$

$\mathrm{V}_{10}=\mathrm{HC}-2$

Interaction effects of variety and salinity level on percent germination was found significant (table 1). Percent germination was gradually decreased with increased salinity level at both seven and fourteen days after sowing. At seven days after sowing highest percent germination from the interaction of Control $\times$ O-72 (92\%) and the lowest was from interaction of Control $\times \mathrm{HC}-2(77 \%)$, besides highest percent germination from the interaction of $100 \mathrm{mM} \times \mathrm{O}$ $795(44 \%)$ and the lowest was from interaction of $100 \mathrm{mM} \times \mathrm{HC}-95$ (22\%). Afterwards, fourteen days after sowing highest percent germination from the interaction of Control $\times$ O-72 $(92 \%)$ and the lowest was from Control $\times$ HC-2 $(77 \%)$, besides highest percent germination from the interaction of $100 \mathrm{mM} \times \mathrm{CVE}-3(11 \%)$ and the lowest was from $100 \mathrm{mM} \times \mathrm{HC}-95(2 \%)$. Salinity stress decrease germination drastically in all of the jute and kenaf varieties. When salinity levels increase then germination inhibition (\%) of both jute and kenaf varieties also increase. A salinity level of $100 \mathrm{mM}$ caused the highest germination inhibition $(74.70 \%)$ in jute variety CVL-1, which was very close to those of BJC-7370 andO-72. On the other hand, the lowest germination inhibition $(51.11 \%)$ was recorded in jute variety O-795. So based on the germination inhibition (\%) under salinity stress, salt tolerant varieties were (7 days after seed sowing)

Plant height

Plant height among the varieties was significant at $1 \%$ level of probability (table 3$)$. Variety CVE-3 $(105.7 \mathrm{~cm})$ produced the tallest plant height followed by C-83 $(94.00 \mathrm{~cm}), \mathrm{O}-72(87.73 \mathrm{~cm}), \mathrm{OM}-1$ $(83.92 \mathrm{~cm})$ and the lowest value was produced by $\mathrm{O}-795(62.13 \mathrm{~cm})$. The results showed that different varieties had different degrees of salt tolerance for plant height.

Number of leaves
From table 3, the maximum number of leaves was found in BJC7370 (17.50) followed by statistically similar to O-72 (17.08). The lowest tiller production was found in HC-95 (12.29). From the results it was evident that different varieties had different levels of salinity tolerance for number of leaves.

Total dry weight

From table 3 , among the varieties, total dry weight was statistically significant. The maximum total dry matter was observed in CVE$3(14.80 \mathrm{gm})$ and the minimum (8.692) was in O-795. It indicates that the tolerance of different cultivars is different in producing of dry weight. In this context, CVE-3 showed more tolerance than in others.

Table 3: Effect of Varieties on Number of Leaves per Plant, Total Dry Weight and Plant Height of Jute and Kenaf

\begin{tabular}{llll}
\hline Variety & $\begin{array}{l}\text { Number of leaves } \\
\text { per plant }\end{array}$ & $\begin{array}{l}\text { Total dry } \\
\text { weight }(\mathrm{g})\end{array}$ & $\begin{array}{l}\text { Plant height } \\
(\mathrm{cm})\end{array}$ \\
\hline CVE-3 & $12.67 \mathrm{e}$ & $14.80 \mathrm{a}$ & $105.7 \mathrm{a}$ \\
C-83 & $14.67 \mathrm{c}$ & $13.11 \mathrm{~b}$ & $94.00 \mathrm{~b}$ \\
CVL-1 & $14.25 \mathrm{~cd}$ & $11.24 \mathrm{de}$ & $80.78 \mathrm{e}$ \\
BJC-7370 & $17.50 \mathrm{a}$ & $10.37 \mathrm{~g}$ & $75.21 \mathrm{~g}$ \\
O-795 & $12.58 \mathrm{e}$ & $8.692 \mathrm{~h}$ & $62.13 \mathrm{~h}$ \\
O-9897 & $15.50 \mathrm{~b}$ & $8.937 \mathrm{~h}$ & $63.79 \mathrm{~h}$ \\
OM-1 & $12.75 \mathrm{e}$ & $11.72 \mathrm{~d}$ & $83.92 \mathrm{~d}$ \\
O-72 & $17.08 \mathrm{a}$ & $12.28 \mathrm{c}$ & $87.73 \mathrm{c}$ \\
HC-95 & $12.29 \mathrm{e}$ & $10.66 \mathrm{fg}$ & $77.50 \mathrm{f}$ \\
HC-2 & $13.67 \mathrm{~d}$ & $11.07 \mathrm{ef}$ & $81.48 \mathrm{e}$ \\
LSD 0.05 & 0.762 & 0.545 & 2.07 \\
Level of signif- & $* *$ & $* *$ & $* *$ \\
icance & & & 3.14 \\
CV\% & 6.56 & 5.95 & \\
\hline
\end{tabular}

In a column, figures with common letters or without letter do not differ significantly, whereas figures with dissimilar letters differ significantly (as per DMRT).

** indicates significant at $1 \%$ level of probability.

Table 4: Interaction Effect of Variety and Salinity Level on Number of Leaves per Plant, Total Dry Weight and Plant Height of Jute and Kenaf

\begin{tabular}{|c|c|c|c|c|c|c|}
\hline Salinity level & $\begin{array}{l}\text { Number of } \\
\text { leaves per } \\
\text { plant }\end{array}$ & $\begin{array}{l}\text { Decrease in number of } \\
\text { leaves }(\%) \text { due to salin- } \\
\text { ity }\end{array}$ & $\begin{array}{l}\text { Total dry } \\
\text { weight }(\mathrm{g})\end{array}$ & $\begin{array}{l}\text { Decrease in Total dry } \\
\text { weight }(\mathrm{g})(\%) \text { due to sa- } \\
\text { linity }\end{array}$ & $\begin{array}{l}\text { Plant } \\
\text { height } \\
(\mathrm{cm})\end{array}$ & $\begin{array}{l}\text { Decrease in Plant height } \\
(\mathrm{cm})(\%) \text { due to salinity }\end{array}$ \\
\hline CVE- $3 \times 0 \mathrm{mM}$ & $22.00 \mathrm{~b}$ & 0 & $20.55 \mathrm{a}$ & 0 & $145.2 \mathrm{a}$ & 0 \\
\hline CVE $-3 \times 25 \mathrm{mM}$ & $14.33 \mathrm{gh}$ & 34.86 & $18.52 \mathrm{~b}$ & 9.87 & $134.8 \mathrm{~b}$ & 7.62 \\
\hline CVE $-3 \times 50 \mathrm{mM}$ & 8.667 no & 60.59 & $12.39 \mathrm{f}$ & 39.70 & $86.98 \mathrm{ij}$ & 40.09 \\
\hline CVE- $3 \times 75 \mathrm{mM}$ & $5.667 \mathrm{q}$ & 74.22 & $7.830 \mathrm{~lm}$ & 61.89 & $56.33 \mathrm{o}$ & 61.20 \\
\hline C- $-83 \times 0 \mathrm{mM}$ & 20.33 cde & 0 & $17.47 \mathrm{bc}$ & 0 & $125.3 \mathrm{~b}$ & 0 \\
\hline $\mathrm{C}-83 \times 25 \mathrm{mM}$ & $17.67 \mathrm{f}$ & 13.08 & $16.67 \mathrm{cde}$ & 4.57 & $119.4 \mathrm{de}$ & 4.70 \\
\hline $\mathrm{C}-83 \times 50 \mathrm{mM}$ & $13.00 \mathrm{hij}$ & 36.05 & $12.61 \mathrm{f}$ & 21.81 & $90.25 \mathrm{hi}$ & 27.97 \\
\hline C- $83 \times 75 \mathrm{mM}$ & 7.667 op & 62.28 & 5.690 ор & 67.42 & $41.08 \mathrm{st}$ & 67.21 \\
\hline CVL- $1 \times 0 \mathrm{mM}$ & $21.33 \mathrm{bcd}$ & 0 & $16.76 \mathrm{~cd}$ & 0 & $120.3 \mathrm{~d}$ & 0 \\
\hline CVL- $1 \times 25 \mathrm{mM}$ & 13.67 ghij & 35.91 & $15.95 \mathrm{de}$ & 4.83 & $114.8 \mathrm{fg}$ & 4.57 \\
\hline CVL- $1 \times 50 \mathrm{mM}$ & $12.33 \mathrm{ijk}$ & 42.19 & $7.730 \mathrm{~lm}$ & 53.87 & $55.83 \mathrm{o}$ & 53.59 \\
\hline CVL- $1 \times 75 \mathrm{mM}$ & $9.667 \mathrm{lmn}$ & 54.67 & $4.410 \mathrm{q}$ & 73.68 & $31.70 \mathrm{v}$ & 73.64 \\
\hline $\begin{array}{l}\text { BJC- } \\
7370 \times 0 \mathrm{mM}\end{array}$ & $25.33 \mathrm{a}$ & 0 & $15.77 \mathrm{de}$ & 0 & $112.5 \mathrm{~g}$ & 0 \\
\hline $\begin{array}{l}\text { BJC- } \\
7370 \times 25 \mathrm{mM}\end{array}$ & $19.67 \mathrm{e}$ & 22.34 & $12.79 \mathrm{f}$ & 18.89 & $91.83 \mathrm{~h}$ & 18.37 \\
\hline $\begin{array}{l}\text { BJC- } \\
7370 \times 50 \mathrm{mM}\end{array}$ & $14.67 \mathrm{gh}$ & 42.08 & $8.380 \mathrm{kl}$ & 46.86 & $61.33 n$ & 45.48 \\
\hline $\begin{array}{l}\text { BJC- } \\
7370 \times 75 \mathrm{mM}\end{array}$ & $10.33 \mathrm{~lm}$ & 59.21 & $4.523 \mathrm{pq}$ & 71.31 & $35.17 \mathrm{uv}$ & 68.73 \\
\hline O- $795 \times 0 \mathrm{mM}$ & 19.00 ef & 0 & $12.39 \mathrm{f}$ & 0 & $87.50 \mathrm{hij}$ & 0 \\
\hline $\mathrm{O}-795 \times 25 \mathrm{mM}$ & $14.00 \mathrm{ghi}$ & 26.31 & $10.20 \mathrm{ij}$ & 17.67 & $73.08 \mathrm{~m}$ & 16.48 \\
\hline O- $795 \times 50 \mathrm{mM}$ & $11.33 \mathrm{kl}$ & 40.36 & $6.943 \mathrm{mn}$ & 43.98 & $49.83 \mathrm{pq}$ & 43.05 \\
\hline O- $795 \times 75 \mathrm{mM}$ & $6.000 \mathrm{pq}$ & 68.42 & $5.237 \mathrm{opq}$ & 57.73 & 38.08 tu & 56.48 \\
\hline O- $9897 \times 0 \mathrm{mM}$ & $24.67 \mathrm{a}$ & 0 & $12.10 \mathrm{fg}$ & 0 & $86.67 \mathrm{ij}$ & 0 \\
\hline O- $9897 \times 25 \mathrm{mM}$ & $17.33 \mathrm{f}$ & 29.75 & $10.62 \mathrm{hi}$ & 12.23 & $75.08 \mathrm{~lm}$ & 13.37 \\
\hline O- $9897 \times 50 \mathrm{mM}$ & 13.33 ghij & 45.96 & $7.090 \mathrm{mn}$ & 41.40 & $50.58 \mathrm{p}$ & 41.64 \\
\hline O- $9897 \times 75 \mathrm{mM}$ & $6.6 \mathrm{pq}$ & 73.24 & 5.933 no & 50.99 & $42.83 \mathrm{rs}$ & 50.58 \\
\hline $\mathrm{OM}-1 \times 0 \mathrm{mM}$ & 18.67 ef & 0 & $16.75 \mathrm{~cd}$ & 0 & $120.3 \mathrm{~d}$ & 0 \\
\hline $\mathrm{OM}-1 \times 25 \mathrm{mM}$ & $14.33 \mathrm{gh}$ & 23.24 & $15.81 \mathrm{de}$ & 5.61 & $114.4 \mathrm{fg}$ & 4.67 \\
\hline $\mathrm{OM}-1 \times 50 \mathrm{mM}$ & $10.67 \mathrm{klm}$ & 42.84 & $9.040 \mathrm{jk}$ & 46.02 & $63.83 n$ & 46.80 \\
\hline $\mathrm{OM}-1 \times 75 \mathrm{mM}$ & $7.333 \mathrm{opq}$ & 60.73 & $5.277 \mathrm{opq}$ & 68.53 & $37.08 \mathrm{tu}$ & 69.10 \\
\hline $\mathrm{O}-72 \times 0 \mathrm{mM}$ & $24.67 \mathrm{a}$ & 0 & $17.52 \mathrm{bc}$ & 0 & $125.25 \mathrm{~b}$ & 0 \\
\hline $\mathrm{O}-72 \times 25 \mathrm{mM}$ & $21.67 \mathrm{bc}$ & 12.16 & $16.46 \mathrm{cde}$ & 6.05 & $118.7 \mathrm{def}$ & 5.26 \\
\hline
\end{tabular}




\begin{tabular}{|c|c|c|c|c|c|c|}
\hline $\mathrm{O}-72 \times 50 \mathrm{mM}$ & $15.00 \mathrm{~g}$ & 39.19 & $8.670 \mathrm{kl}$ & 50.51 & $60.83 n$ & 51.45 \\
\hline $\mathrm{O}-72 \times 75 \mathrm{mM}$ & $7.000 \mathrm{pq}$ & 71.62 & 6.473 no & 63.05 & $46.08 \mathrm{pqr}$ & 63.22 \\
\hline $\mathrm{HC}-95 \times 0 \mathrm{mM}$ & $17.80 \mathrm{f}$ & 0 & $15.48 \mathrm{e}$ & 0 & $110.7 \mathrm{~g}$ & 0 \\
\hline HC $-95 \times 25 \mathrm{mM}$ & $14.36 \mathrm{gh}$ & 19.32 & $11.06 \mathrm{ghi}$ & 28.55 & $79.58 \mathrm{k}$ & 28.11 \\
\hline HC- $95 \times 50 \mathrm{mM}$ & $11.20 \mathrm{klm}$ & 37.07 & $9.963 \mathrm{ij}$ & 35.65 & $74.33 \mathrm{~lm}$ & 32.85 \\
\hline HC- $-95 \times 75 \mathrm{mM}$ & $5.800 \mathrm{q}$ & 67.41 & 6.150 no & 60.27 & 45.42 qrs & 58.97 \\
\hline $\mathrm{HC}-2 \times 0 \mathrm{mM}$ & $19.83 \mathrm{de}$ & 0 & $16.28 \mathrm{cde}$ & 0 & $115.2 \mathrm{efg}$ & 0 \\
\hline $\mathrm{HC}-2 \times 25 \mathrm{mM}$ & $13.20 \mathrm{hij}$ & 33.43 & $11.72 \mathrm{fgh}$ & 28.00 & $84.75 \mathrm{j}$ & 26.43 \\
\hline $\mathrm{HC}-2 \times 50 \mathrm{mM}$ & $12.06 \mathrm{jk}$ & 40.19 & $10.17 \mathrm{ij}$ & 37.53 & $78.42 \mathrm{kl}$ & 31.92 \\
\hline $\mathrm{HC}-2 \times 75 \mathrm{mM}$ & $9.583 \mathrm{mn}$ & 51.68 & 6.110 no & 62.46 & $47.58 \mathrm{pq}$ & 58.69 \\
\hline $\mathrm{LSD}_{0.05}$ & 1.53 & & 1.09 & & & \\
\hline $\begin{array}{l}\text { Level of signifi- } \\
\text { cance }\end{array}$ & $* *$ & & $* *$ & & $* *$ & \\
\hline $\mathrm{CV} \%$ & 6.56 & & 5.95 & & 3.14 & \\
\hline
\end{tabular}

In a column, figures with common letters or without letter do not differ significantly, whereas figures with dissimilar letters differ significantly (as per DMRT).

** indicates significant at $1 \%$ level of probability.

\section{Conclusion}

A study was conducted in the seed laboratory of agronomy, Bangladesh Agricultural University, Mymensingh from the period 15 April 2012 to 30 April 2012 for evaluating the rate of germination (percentage) of jute and kenaf varieties for salinity tolerance. Germination of seeds of eight jute and two kenaf varieties were evaluated under six salinity levels $0 \mathrm{mM}, 20 \mathrm{mM}, 40 \mathrm{mM}, 60 \mathrm{mM}, 80 \mathrm{mM}$, and $100 \mathrm{mM} \mathrm{NaCl}$ in the seed laboratory of the Department. The results from the germination study revealed that under control condition $(0 \mathrm{mM} \mathrm{NaCl})$ all the jute varieties showed germination more than $80 \%$ both at 7 and 14 days after seed sowing, whereas kenaf varieties had germination a little less than $80 \%$. Among the jute varieties, O-72 showed the highest germination (92\%), which was statistically similar with those of OM-1(91\%), O-795 (90\%), and C$83(87 \%)$ and two kenaf varieties HC-2 showed the highest germination $(53.50 \%)$ and remaining kenaf variety HC-95 shows germination $(48.50 \%)$. The percentage of germination decreased with increasing salinity levels in all of the jute and kenaf varieties. A salinity level of $100 \mathrm{mM}$ caused the highest germination inhibition $(74.70 \%)$ in jute variety CVL-1, which was very close to those of BJC-7370 and O-72. On the other hand, the lowest germination inhibition $(51.11 \%$ ) was recorded in jute variety O-795. Again in kenaf varieties the highest germination inhibition HC-95 (72.22\%) and the lowest one were recorded from the variety HC-2 $(61.03 \%)$. Based on the germination inhibition (\%) O-795 shows lowest germination inhibition $(51.11 \%)$ at 7 days of seed sowing. On the other hand, O-9897 in jute variety shows the lowest germination inhibition $(86.74 \%)$ closest to those of CVE-3 (86.90\%), C-83 (88.50\%), and BJC-7370 (89.53\%) at 14 days of seed sowing. So based on the study it can be suggested that use of O-9897 variety of jute shows the lowest germination inhibition (\%) after 14 days of seed sowing in $100 \mathrm{mM}$ salinity level condition can tolerate saline condition comparatively all other varieties of jute and kenaf would be a promising practice to maximize germination.

\section{Acknowledgement}

Special Thanks to Dr. Abdul Kader, Professor, Department of Agronomy Bangladesh Agricultural University for his suggestions, guidance, and cooperation.

\section{References}

[1] Akhtaruzzaman, A. F. M. and Shafi M 1995. Pulping of jute.Tappi J., 78(2): 106-112.

[2] Alim A. 1978. A Hand Book of Bangladesh Jute. Associated Printer Ltd., 311 Johnson Road, Dhaka.p. 10.

[3] Greenway H, Munns R 1980. Mechanism of salt tolerance in nonhalophytes.Ann. Rev. Plant Physiol., 31,149-190 https://doi.org/10.1146/annurev.pp.31.060180.001053.
[4] Gufran K. M. 1994. Effect of salt stress on nitrate reductage activity in some leguminous crops. Indian J. Plant Physiol., 37, 185-187.

[5] Jahan, M. S. 2001. Evaluation of additive in soda pulping of jute. Tappi J., 84(8): 1-11.

[6] Ma, H. Y., R... J. Wang, X. S. Wang and H. 2009. Identification and evaluation of salt tolerance of jute germplasm during germination and seedling periods. Journal of plant genetic resources, 10(2): 236243. (in Chinese)

[7] Purhpan R and Rangasamy S. R. S. 2002. In vivo response of jute cultivars to salt strees. J. Ecobiology. 14(3), 177-182.

[8] Saha 1996. Effect of intercropping mustard, corriander, red amaranth and radish with late jute seed rate. MS Thesis, Dept. Agron., Bangladesh Agril. Univ., Mymensingh.

[9] Singh R. A., Roy N. K., and Hoque M. S. 2001. Changes in growth and metabolic activity in seedlings of lentil (Lens culinaris Medie) genotypes during salt stress. Indian J. Plant Physiol., 6, 406-410.

[10] Varshney K. A., Sanwal N, Agarwl N. 1998. Salinity induced changes in ion uptake and chemical composition in chickpea (CicerarietinumL.). Indian J. Plant Physiol., 3, 140-142.Waisel Y (1972) Biology of Halophytes. Aca. Press, New York. 11, 1-14.

[11] UNDP and FAO (United Nations Development Programme and Food and Agriculture Organization). 1988. Land Resources Appraisal of Bangladesh For Agricultural Development Report No. 2. Agroecological Regions of Bangladesh.United Nations Dev. Prog. \& Food and Agric. Org. United Nations. pp. 211-212. 